\title{
A NOTE ON QUASI-ASSOCIATIVE ALGEBRAS
}

\author{
KEVIN MCCRIMMON ${ }^{1}$
}

By "algebra" we will mean nonassociative algebra with identity over a field $\Phi$ of characteristic $\neq 2$. We do not assume finite dimensionality. If $\mathfrak{A}=(\mathfrak{X}, \cdot)$ is an algebra on a vector space $\mathfrak{X}$ and $\lambda \in \Phi$ then the $\lambda$-mutation of $\mathfrak{A}$ is

$$
\mathfrak{A}^{(\lambda)}=\left(\mathfrak{X},{ }_{\lambda}\right)
$$

where a new multiplication on $\mathfrak{X}$ is introduced by

$$
x \cdot{ }_{\lambda} y=\lambda x \cdot y+(1-\lambda) y \cdot x .
$$

A mutation $\mathfrak{D}^{(\lambda)}$ of an associative algebra for $\lambda \neq 1 / 2$ is called a split quasi-associative algebra. An algebra $\mathfrak{A}$ is called quasi-associative if it is a form of a split quasi-associative algebra, i.e., there is a splitting field $\Omega \supset \Phi$ and $\lambda \in \Omega, \lambda \neq 1 / 2$ such that the extension $\mathfrak{A}_{\Omega}$ is a $\lambda$-mutation of an associative algebra $\mathfrak{D}$ over $\Omega$ :

$$
\mathfrak{A}_{\Omega}=\mathfrak{D}^{(\lambda)} \text {. }
$$

Quasi-associative algebras play an important role in the theory of nonassociative algebras [1, pp. 581-584]; [2, pp. 192-193]. Although the split quasi-associative algebras have a natural representation, the nonsplit quasi-associative algebras have always seemed to need a similar treatment. It is the purpose of this note to relate them in a natural way to associative algebras, Jordan algebras, Lie algebras, and involutions.

The idea is roughly the following. If $\mathfrak{A}$ is a nonsplit quasi-associative algebra we will find a split quasi-associative algebra $\mathfrak{D}^{(\lambda)}$ (for $\mathfrak{D}$ associative) with an automorphism * such that $\mathfrak{A}$ is the subalgebra of fixed points:

$$
\mathfrak{U}=\mathfrak{S}\left(\mathfrak{D}^{(\lambda)},{ }^{*}\right) .
$$

The automorphism * of $\mathfrak{D}^{(\lambda)}$ will at the same time be an involution on D. Conversely, if $\mathfrak{D}$ is an associative algebra with an involution * and a suitable element $\lambda$ in its center then

$$
\mathfrak{U}=\mathfrak{S}\left(\mathfrak{S}^{(\lambda)}, *\right)
$$

will be a nonsplit quasi-associative algebra.

This correspondence between nonsplit quasi-associative algebras

Received by the editors May 23, 1966.

1 This research was supported by an AFOSR Postdoctoral Fellowship. 
and associative algebras with involution depends on the scalar $\lambda$ and the associative algebra $\mathfrak{D}$, and these are not uniquely determined by the representation (2). Indeed, if $\mathfrak{D}^{*}$ is the opposite algebra (antiisomorphic) to $I$ then

$$
\mathfrak{D}^{(\lambda)}=\mathfrak{D}^{*(1-\lambda)} .
$$

To obtain a natural one-to-one correspondence we must consider not only nonsplit quasi-associative algebras $\mathfrak{U}$ and associative algebras $(D)$ with involution ${ }^{*}$ but also a choice of $\lambda$; what we obtain is a correspondence between pairs $(\mathfrak{A}, \lambda)$ and triples $(\mathfrak{D}, *, \lambda)$.

Instead of $\lambda$ it will be technically more convenient to work with

$$
\Delta=2 \lambda-1 \text {. }
$$

The square of this element

$$
\delta=\Delta^{2}
$$

is called the discriminant of the algebra $\mathfrak{U}$ and is an element of the base field $\Phi$ intrinsically determined by $\mathfrak{Y}$. Indeed, we have the following interesting characterization of quasi-associative algebras.

THEOREM. If $\mathfrak{A}$ is a quasi-associative algebra there exists a nonzero element $\delta \in \Phi$ such that the associators $[x, y, z]$ in $\mathfrak{A}$ and $[x, y, z]+$ in $\mathfrak{A}^{+}$ are related by

$$
[x, y, z]=(1-\delta)[x, y, z]^{+} .
$$

Conversely, if $\mathfrak{A}$ is an algebra in which (6) holds for some $\delta \neq 0$ then $\mathfrak{A}$ is quasi-associative.

If we agree to set $\delta=1$ in case $\mathfrak{A}$ itself is associative then $\delta$ is uniquely determined by $\mathfrak{A}$, and $\mathfrak{A}$ is a split quasi-associative algebra if and only if $\delta \in \Phi^{2}$ is a square in $\Phi$.

Proof. If $\mathfrak{B}$ is an arbitrary algebra and we use (1) to compute the associators $[x, y, z]^{(\lambda)}$ in $\mathfrak{B}^{(\lambda)},[x, y, z]$ in $\mathfrak{B}$, and $[x, y, z]+$ in $\mathfrak{B}^{+}$we obtain the relation

$$
\begin{aligned}
& {[x, y, z]^{(\lambda)}=\lambda(2 \lambda-1)[x, y, z]+(1-\lambda)(2 \lambda-1)[z, y, x]} \\
& +4 \lambda(1-\lambda)[x, y, z]^{+} \text {. }
\end{aligned}
$$

In particular, if $\mathfrak{B}=\mathfrak{D}$ is associative and $\mathfrak{A}$ is quasi-associative as in (2) then (7) reduces to (6) with $\delta$ defined by (4) and (5). This also shows that $\delta \in \Phi$ is uniquely determined if $\mathfrak{A}$ is not associative since we can find elements in $\mathfrak{A}$ for which the left side of (6) does not vanish.

Conversely, suppose (6) is satisfied for some $\delta \neq 0$ and let $\mu$ be a root of the quadratic equation $4 \mu(1-\mu)=1-\delta^{-1}$ in an extension field 
$\Omega=\Phi(\mu)$. Applying (7) with $\lambda, \mathfrak{B}$ replaced by $\mu, \mathfrak{A}_{\Omega}$ and using the hypothesis (6) together with the observation that $[z, y, x]^{+=}$ $-[x, y, z]+$ by commutativity, we wind up with the formula

$$
\begin{gathered}
{[x, y, z]^{(\mu)}=\{\mu(2 \mu-1)(1-\delta)-(1-\mu)(2 \mu-1)(1-\delta)} \\
+4 \mu(1-\mu)\}[x, y, z]^{+}
\end{gathered}
$$

for the associator in $\mathfrak{A}_{\Omega}^{(\mu)}$. Now by construction

$$
\begin{aligned}
\mu(2 \mu-1) & =\frac{1}{2}\left(2 \mu-1+\delta^{-1}\right), \\
(1-\mu)(2 \mu-1) & =\frac{1}{2}\left(2 \mu-1-\delta^{-1}\right), 4 \mu(1-\mu)=\left(1-\delta^{-1}\right) ;
\end{aligned}
$$

so the term in braces is

$$
\delta^{-1}(1-\delta)+\left(1-\delta^{-1}\right)=0
$$

and hence $\mathfrak{D}=\mathfrak{A}_{\Omega}^{(\mu)}$ is associative. The general transitivity relation

$$
\left\{\mathfrak{A}^{(\lambda)}\right\}^{(\mu)}=\mathfrak{A}^{\left(\lambda \odot^{\mu)}\right.}, \quad \lambda \odot \mu=1-\lambda-\mu+2 \lambda \mu
$$

for mutations then implies

as in (2) for

$$
\mathfrak{A}_{\Omega}=\mathfrak{A}_{\Omega}^{(1)}=\mathfrak{A}_{\Omega}^{(\mu \odot \lambda)}=\left\{\mathfrak{U}_{\Omega}^{(\mu)}\right\}^{(\lambda)}=\mathfrak{D}^{(\lambda)}
$$

$$
\lambda=\mu /(2 \mu-1) \quad\left(\text { note } \delta \neq 0 \Rightarrow \mu \neq \frac{1}{2}\right),
$$

and $\mathfrak{A}$ is quasi-associative.

Finally, if $\mathfrak{A}$ is split the discriminant is $1 \in \Phi^{2}$ if $\mathfrak{A}$ is associative and $\Delta^{2} \in \Phi^{2}$ by (4) and (5) otherwise. Conversely, if $\delta \in \Phi^{2}$ then either $\delta=1$, in which case $\mathfrak{A}$ is associative and trivially split, or else $\Delta$ and $\lambda$ are in $\Phi$ by (4) and (5). In this case $\mu=\lambda /(2 \lambda-1)$ is also in $\Phi$, hence $\Omega=\Phi(\mu)=\Phi$ and by the above $\mathfrak{U}=\mathfrak{A}_{\Omega}=\mathfrak{D}^{(\lambda)}$ is split.

The actual correspondence mentioned in the introduction is constructed as follows. To a pair $(\mathfrak{A}, \Delta)$ where $\mathfrak{A}$ is a nonsplit quasiassociative algebra and $\Delta$ a particular square root of its discriminant $\delta$ we associate a triple $\left(\mathfrak{D},{ }^{*}, \Delta\right)$ consisting of an algebra $\mathfrak{D}$, a linear map ${ }^{*}$ of period 2 on $\mathfrak{D}$, and an element $\Delta$ in the center of $\mathfrak{D}$. The algebra $\mathfrak{D}$ is defined as the mutation of a certain extension of $\mathfrak{A}$ :

$$
\mathfrak{D}=\mathfrak{A}_{\Omega}^{(\mu)} \quad \text { where } \Omega=\Phi(\Delta), \mu=\frac{1}{2}\left(1+\Delta^{-1}\right) .
$$

Since $\Omega$ is a quadratic field, an automorphism * of $\Omega=\Phi+\Phi \Delta$ is completely determined by taking $\Delta^{*}=-\Delta$, and this extends to an automorphism $* \otimes 1$ of $\mathfrak{A}_{\Omega}=\Omega \otimes \mathfrak{A}$ :

$$
(\omega \otimes a)^{*}=\omega^{*} \otimes a, \quad(\alpha+\beta \Delta)^{*}=\alpha-\beta \Delta
$$

$$
\text { for } \omega \in \Omega, a \in \mathfrak{A}, \alpha, \beta \in \Phi \text {. }
$$


The element in the center of $\mathfrak{D}$ is just a scalar multiple of the identity:

$$
\Delta=\Delta 1 \text {. }
$$

In the reverse direction, given an associative algebra $\mathfrak{D}$ with involution ${ }^{*}$ and an element $\Delta$ of the center with $\Delta^{*}=-\Delta, \Delta^{2} \in \Phi 1$, $\Delta^{2} \notin \Phi^{2} 1$ we associate to the triple $(\mathfrak{D}, *, \Delta)$ a pair ( $\left.\mathfrak{A}, \Delta\right)$ consisting of an algebra $\mathfrak{A}$ and an element $\Delta$ in an extension field of $\Phi$. The algebra $\mathfrak{A}$ is defined as the subalgebra of a certain mutation of $\mathfrak{D}$ left fixed by *:

$$
\mathfrak{A}=\mathfrak{S}\left(\mathfrak{D}^{(\lambda)}, *\right) \quad \text { where } \lambda=\frac{1}{2}(1+\Delta) .
$$

The assumptions about $\Delta$ insure that it belongs to a quadratic extension $\Omega=\Phi(\Delta) \subset \mathfrak{D}$ :

$$
\Delta 1=\Delta .
$$

THEOREM. The correspondence set up by (9) and (10) is a natural one-to-one correspondence

$$
(\mathfrak{H}, \Delta) \leftrightarrow(\mathfrak{I}, *, \Delta)
$$

between the nonsplit quasi-associative algebras $\mathfrak{H}$ with discriminant $\Delta^{2}$ and the associative algebras $\mathfrak{D}$ with involution * which satisfy $[\mathfrak{D},[\mathfrak{D}, \mathfrak{D}]]$ $\neq 0$ and which contain an element $\Delta$ in their center satisfying $\Delta^{*}=-\Delta$, $\Delta^{2} \in \Phi 1, \Delta^{2} \notin \Phi^{2} 1$.

Under this correspondence the Jordan algebra of *-symmetric elements of $\mathfrak{D}$ is just the symmetrized algebra of $\mathfrak{A}$

$$
\mathfrak{A}^{+}=\mathfrak{S}\left(\mathfrak{D},{ }^{*}\right)
$$

and the Lie algebra of ${ }^{*}$-skew elements is isomorphic to the skew algebra of $\mathfrak{A}$

$$
\mathfrak{H}^{-} \cong \mathfrak{S}\left(\mathfrak{D},{ }^{*}\right) \text {. }
$$

Proof. First assume we are given ( $(A, \Delta)$. Then (6) holds for $\delta=\Delta^{2}$, and in the proof of the first theorem we saw that $\mathfrak{D}=\mathfrak{P}_{\Omega}^{(\mu)}$ is associative since $4 \mu(1-\mu)=\left(1+\Delta^{-1}\right)\left(1-\Delta^{-1}\right)=1-\Delta^{-2}=1-\delta^{-1}$. Since ${ }^{*}$ is an automorphism of $\mathfrak{I}_{\Omega}$ and $\mu^{*}=1-\mu$ we see that for $x, y \in \mathfrak{D}=\mathfrak{A}_{\Omega}^{(\mu)}$

$$
(x y)^{*}=\left(x \cdot{ }_{\mu} y\right)^{*}=x^{*} \cdot{ }_{\mu^{*}} y^{*}=x^{*} \cdot{ }_{1-\mu} y^{*}=y^{*} \cdot{ }_{\mu} x^{*}=y^{*} x^{*}
$$

and $*$ is an involution on $\mathfrak{D}$. Clearly $\Delta 1$ is an element of the center satisfying $\Delta^{*}=-\Delta, \Delta^{2}=\delta 1 \in \Phi 1$, and $\Delta^{2} \notin \Phi^{2} 1$ by the first theorem since $\mathfrak{P}$ is nonsplit. Finally, we have the formula

$$
4\lfloor x, y, z]^{+}=[y,[x, z]]
$$

in an associative algebra $\mathfrak{D}$. Since $\mathfrak{A}$ is not associative and associators 
in $\mathfrak{P}^{+}$and $\mathfrak{D}^{+}$coincide, (6) shows $[\mathfrak{D},[\mathfrak{D}, \mathfrak{D}]] \neq 0$. Thus the triple $(\mathfrak{D}, *, \Delta)$ defined by $(9 \mathrm{a}),(9 \mathrm{~b}),(9 \mathrm{c})$ is of the required type.

Conversely, suppose we are given $(\mathfrak{D}, *, \Delta)$. Then $\lambda=\frac{1}{2}(1+\Delta)$ has $\lambda^{*}=1-\lambda$, so the fact that ${ }^{*}$ is an involution on $\mathfrak{D}$ implies

$$
\left(x \cdot{ }_{\lambda} y\right)^{*}=y^{*} \cdot{ }_{\lambda *} x^{*}=y^{*} \cdot{ }_{1-\lambda} x^{*}=x^{*} \cdot{ }_{\lambda} y^{*}
$$

and ${ }^{*}$ is an automorphism of $\mathfrak{D}^{(\lambda)}$. Thus $\mathfrak{A}=\mathfrak{S}\left(\mathfrak{D}^{(\lambda)},{ }^{*}\right)$ is an algebra. Since $\Delta$ is a skew element of the center we have $\mathfrak{D}^{(\lambda)}=\mathfrak{S}\left(\mathfrak{D}^{(\lambda)},{ }^{*}\right)$ $+\mathfrak{S}\left(\mathfrak{D}^{(\lambda)},{ }^{*}\right)=\mathfrak{A}+\mathfrak{A} \Delta=\mathfrak{A}_{\Omega}$ and $\Omega=\Phi(\Delta)$ is a splitting field for $\mathfrak{A}$. Since $\delta \neq 1$ and $[\mathfrak{P},[\mathfrak{D}, \mathfrak{D}]] \neq 0$ we see by $(13)$ and $(6)$ that $\mathfrak{A}$ is not associative, so it is quasi-associative with discriminant $\delta$. By the first theorem, $\delta \notin \Phi^{2}$ implies $\mathfrak{A}$ is nonsplit. Thus the pair ( $\mathfrak{A}, \Delta$ ) defined by $(10 \mathrm{a}),(10 \mathrm{~b})$ is of the required type.

The correspondence $(\mathfrak{A}, \Delta) \rightarrow\left(\mathfrak{D},{ }^{*}, \Delta\right) \rightarrow(\mathfrak{A}, \Delta)$ is the identity since the resulting algebra is $\mathfrak{S}\left(\mathfrak{D}^{(\lambda)},{ }^{*}\right)$ where $\mathfrak{D}=\mathfrak{H}_{\Omega}^{(\mu)}$, so by $(8) \mathfrak{D}^{(\lambda)}=\mathfrak{A}_{\Omega}$, and hence by $(9 \mathrm{~b}) \mathfrak{S}\left(\mathfrak{A}_{\Omega},{ }^{*}\right)=\mathfrak{A}$. Going the other way, the correspondence $\left(\mathfrak{D},{ }^{*}, \Delta\right) \rightarrow(\mathfrak{A}, \Delta) \rightarrow(\mathfrak{D}, *, \Delta)$ is the identity since the resulting algebra is $\mathfrak{A}_{\Omega}^{(\mu)}$ where we saw that $\mathfrak{U}=\mathfrak{S}\left(\mathfrak{D}^{(\lambda)},{ }^{*}\right)$ has $\mathfrak{A}_{\Omega}=\mathfrak{D}^{(\lambda)}$ and hence by (8) $\mathfrak{H}_{\Omega}^{(\mu)}=\mathfrak{D}$, and since the resulting involution on $\mathfrak{I}_{\Omega}=\Omega \otimes \mathfrak{U}$ agrees with ${ }^{*}$ on $\mathfrak{U}$ (both are the identity) and on $\Omega\left(\Delta^{*}=-\Delta\right)$. Thus the correspondence $(\mathfrak{A}, \Delta) \leftrightarrow(\mathfrak{D}, *, \Delta)$ is one-to-one.

To prove (11) we note that both sides consist of the ${ }^{*}$-symmetric elements with the multiplication induced by $\mathfrak{D}^{(\lambda)+}=\mathfrak{D}^{+}$. To prove (12), we have noted that $\subseteq\left(\mathfrak{D},{ }^{*}\right)=\mathfrak{H} \Delta$, and the map $a \rightarrow a \Delta$ is an isomorphism of $\mathfrak{A}-$ onto $\mathfrak{A} \Delta$ because of the formula $[x, y]=[x, y]_{\mathbb{D} \Delta}$ relating the commutator in $\mathfrak{A}$ with that in $\mathfrak{D}$ by means of (1) and (2).

In closing, we remark that the theorem reduces the classification of simple nonsplit quasi-associative algebras to the classification of simple associative algebras with involutions of the second kind. In fact, the simple nonsplit quasi-associative algebras are precisely the $\mathfrak{S}\left(\mathfrak{D}^{(\lambda)},{ }^{*}\right)$ for simple associative $\mathfrak{D}$ with $[\mathfrak{D},[\mathfrak{D} \mathfrak{D}]] \neq 0,{ }^{*}$ an involution of the second kind, and $\lambda \neq \frac{1}{2}$ an element of the center such that $\lambda+\lambda^{*}=1$. Two such algebras $\mathfrak{S}\left(\mathfrak{D}^{(\lambda)},{ }^{*}\right)$ and $\mathfrak{S}\left(\aleph^{(\mu)},{ }^{*}\right)$ are isomorphic if and only if either $\mathfrak{D}$ is isomorphic to $\mathbb{E}$ and $\lambda=\mu$ or $\mathfrak{D}$ is anti-isomorphic to $\mathbb{E}$ and $\lambda=1-\mu$. (These are just the cases suggested by (3).)

\section{REFERENCES}

1. A. A. Albert, Power associative rings, Tran. Amer. Math. Soc. 64 (1948), 552593.

2. K. McCrimmon, Structure and representations of noncommutative Jordan algebras, Trans. Amer. Math. Soc. 121 (1966), 187-199. 